\section{Anchor effects on the discrimination of pitch*}

\author{
ROBERT ADAMSON \\ Florida Atlantic University, Boca Raton, Florida 33432
}

Two experiments considered the effects of introducing an extreme stimulus (anchor) upon the differential perception of tonal stimuli. In the first experiment, in which Ss rated series stimuli from 1,000 to $2,000 \mathrm{~Hz}$ or from 2,000 to $3,000 \mathrm{~Hz}$, the presence of a $750-\mathrm{Hz}$ anchor apparently disrupted discrimination. A second experiment involved testing for a difference threshold (method of limits) in which the standard was a tone of $1,500 \mathrm{~Hz}$. The uncertainty interval (Iu) was larger when the interval between paired stimulus presentations was filled with a $750-\mathrm{Hz}$ tone, again suggesting that discrimination is impaired by the introduction of a low-frequency anchor. Results are discussed in terms of theories relating range extent and discriminability.

Anchor effects (AE), for this study, are measured in terms of the modification of judgments made about a series of stimuli as the result of the presence of a stimulus value which falls outside the series. One conjecture about such effects is that they are limited by the distance of the anchor from the series stimuli. A further conjecture is that such limits may define relevance between an anchor value and a particular set of stimuli, possibly leading to description of stimulus dimensions in terms of a minimal number of relevant subsets (e.g., Adamson, 1967; Bevan \& Pritchard, 1963; Sarris, 1969).

This study began with an attempt to define $\mathrm{AE}$ limits for a portion of the auditory frequency dimension, itself chosen as passibly anomalous when compared to other quantitative dimensions (see Attneave \& Olsen, 1971). The singular findings of Experiment 1, however, prompted a change in direction and necessitated running a second experiment, in an attempt to ascertain whether the findings were related to basic properties of the perception of pitch.

\section{EXPERIMENT 1}

The purpose of Experiment 1 was to investigate the effect of a constant anchor stimulus upon judgments made about two sets of series stimuli, the conjecture being that $\mathrm{AE}$ for the more remote set would be relatively less, possibly approaching a control (nonanchor) function.

\section{Procedure}

Twenty-six upper-division students at Florida Atlantic University, comprising two groups of 13 each,

*This research was supported by the $A$ ir Force Office of Scientific Research (AFSC) U.S. Air Force, Grant AFOSR-69-1823. The writer would like to thank Carol Freeman for her assistance in gathering data. served as Ss. None had any known hearing defects. Each $\mathbf{S}$ rated a series of five tones in terms of relative pitch. The tones were randomly presented over 120 trials, each tone coming on for 2 sec, with an 8-sec interval between presentations. The tones were programmed by a tape programmer, which selected one of six external tuning capacitors wired through a Hewlett-Packard 200C oscillator. Ss were seated in a sound-attenuated chamber (IAC Model 401A).

The Ss rated the tones by turning a dial-pointer, which in turn generated a potentiometric record, divided into 14 equal steps between extreme dial settings. The dial had markings, "very, very low" and "very, very high," only at its extremes. Thus, the responses were bounded, but not category constrained.

Group I initially rated a series of five tones according to the above procedure. The tones were 1,000 , $1,250,1,500,1,750$, and $2,000 \mathrm{~Hz}$ This provided a control measure of pitch judgment. After 1 week, each $S$ returned for a second session, in which an anchor stimulus of $750 \mathrm{~Hz}$ was introduced on every fourth presentation, the other tones remaining the same. The anchor was judged along with the series stimuli. The order, control series-anchor series, was not counterbalanced, since a reverse order might have introduced residual effects from the anchor stimuli, thereby confounding the control.

Group II repeated the procedure, except that the series tones were $2,000,2,250,2,500,2,750$, and $3,000 \mathrm{~Hz}$. The anchor remained at $750 \mathrm{~Hz}$. Thus, the effect was to increase the distance of the anchor from the series stimuli.

\section{RESULTS}

Figure 1 combines the performances of Groups I and II. In both cases, the presence of the anchor stimulus markedly influenced the distribution of judgments, as compared to the control functions. For Group I, the anchor elevated the mean of all judgments from 9.52 for the control condition to 10.53 for the anchor $[F(1,12)=13.83, p<.01]$. Equally pronounced, however, was the curtailment of the range of judgments under the anchor condition. In terms of percentage of possible range, Ss under the control condition used 62.57\%; under the anchor condition, they used only $15 \%[\mathrm{t}(12)=8.46$, $p<.01]$. The difference between the two functions was highly significant, as seen particularly in the Condition by Frequency interaction $[F(4,48)=$ $23.36, \mathrm{p}<.01]$.

For Group II, the mean of all judgments for the control condition was 10.36 and 10.80 for the anchor $[F(1,12)=1.63, p>.05]$. Under the control condition, Ss used $46.1 \%$ of the possible range; under the anchor condition, they used $14.29 \%[\mathrm{t}(12)=$ $6.26, p<.011$. Again, the difference between control and anchor functions was highly significant, the Condition by Frequency interaction $F(4,48)=$ 46.28, $\mathrm{p}<.01$. Thus, the findings concerning Group I were repeated in terms of the interaction and range constriction, but the main effect on mean judgments was not significant for Group II.

Table 1 compares the variability of individual judgments under control and anchor conditions for both groups. For Group I, the presence of the anchor stimulus increased the mean standard deviation from 1.37 (control) to 3.69 (anchor) $[F(1,48)=$ 37.53, $p<.011$. For Group II, the effects are similar, the mean standard deviation being 1.70 (control) and 2.77 (anchor) $[F(1,48)=43.8$, $p<.011$. Judgments of the anchor stimulus, itself, show a mean judgment for Group I of $10.13(\mathrm{SD}=4.5)$, and for Group II, of 10.08 (SD = 3.4).

In summary, the introduction of the anchor stimulus $(750 \mathrm{~Hz}$ ) appeared to diminish judged differences between the series stimuli. The effect was more pronounced for series stimuli close to the anchor $(1,000-2,000 \mathrm{~Hz})$ than for more remote stimuli $(2,000-3,000 \mathrm{~Hz})$, but was still significant for the latter series.

The relative elevation of mean judgments under the anchor condition for Group I as opposed to Group II offers support for the hypothesis. However, the primary finding of the experiment showed that, for both groups, a pronounced AE existed, in terms of producing a relative lack of discrimination for the series stimuli when the anchor was present. This lack of discriminability under the 


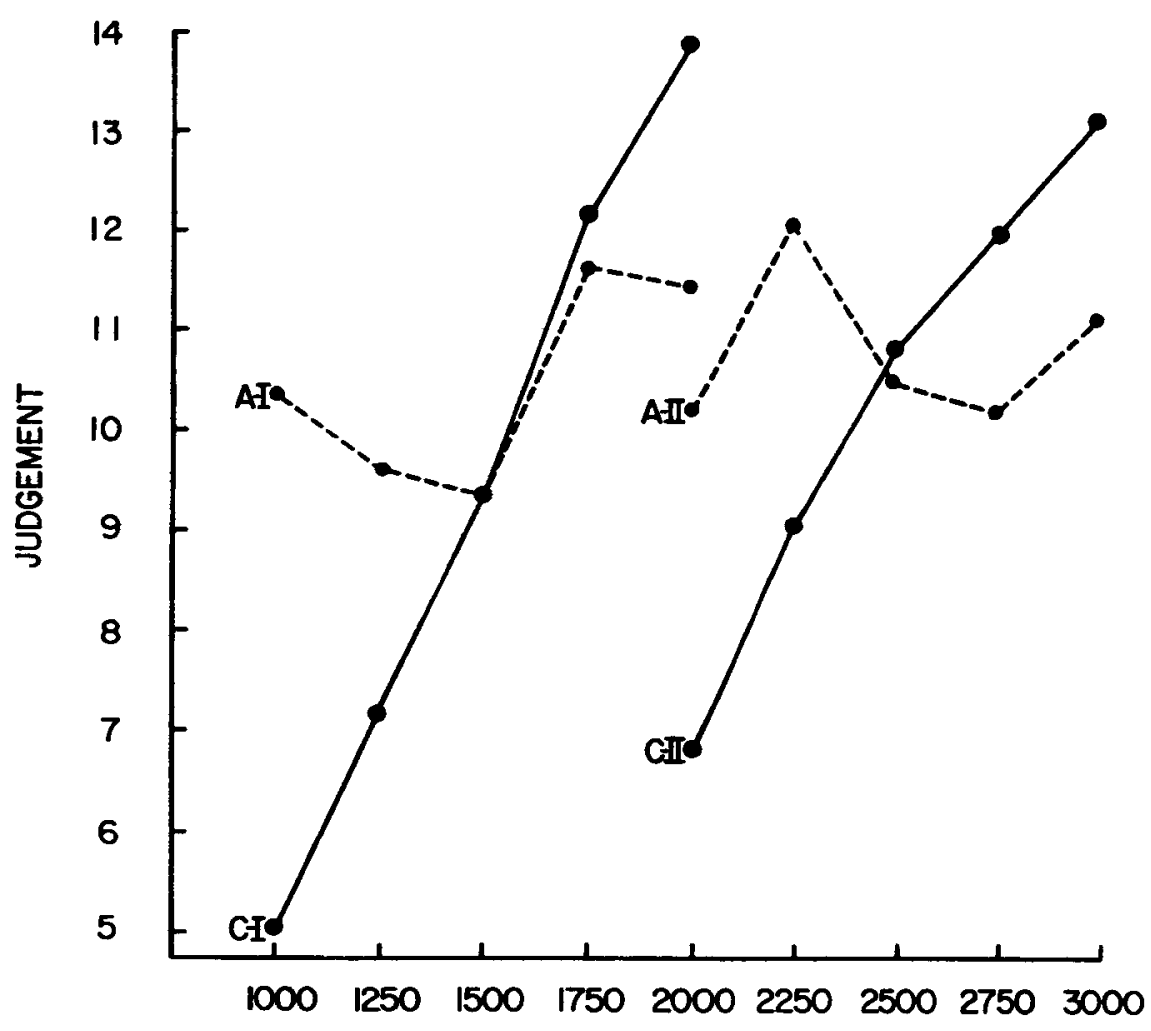

FREQUENCY $(\mathrm{Hz})$

Fig. 1. Series judgments for Groups I and II under control (no-anchor) and anchor (750-Hz tone) conditions.

anchor condition was shown by the decreased range of judgments, the relative lack of slope for the judgment function, and the increased variability of judgments about each stimulus.

\section{EXPERIMENT 2}

The salient question arising from the results of the first experiment is whether or not they reflected simply a response constraint; e.g., Ss could have imposed their own semantic category of "high" on series stimuli relative to the anchor, thereby artificially constricting the possible range of judgments. This conclusion is not supported by the fact that the higher frequency tones were judged higher when presented without the anchor, but the question could persist.

Accordingly, the second experiment attempted to remove possible semantic or response biases and to see if the effect of anchor tones on the perception of pitch still persisted. The argument takes the results of Experiment 1 at face value and states that the introduction of extreme tonal stimuli lessens the perceptual discriminability of other stimuli on the same physical dimension. More specifically, the hypothesis was that in a difference threshold (DL) determination (method of limits), the introduction of a low-tone anchor would increase the interval of uncertainty (Iu: the difference between the upper and lower DLs), this measure bearing an inverse relation to discrimination.

\section{Procedure}

Eleven upper-division students at Florida Atlantic University judged comparison stimuli ranging from 1,450 to $1,550 \mathrm{~Hz}$ against a standard stimulus of $1,500 \mathrm{~Hz}$. The comparison stimuli were presented in randomly ordered ascending and descending series in steps of $5 \mathrm{~Hz}$, except that in the midportion of the range $(1,485-1,515 \mathrm{~Hz})$, changes of $2.5 \mathrm{~Hz}$ were used. Two Hewlett-Packard 200C oscillators were used for stimulus presentation, while a third was used to generate a $750-\mathrm{Hz}$ tone in a portion of the study to be described. All oscillators were voltage-regulated and calibrated with a Hamner scaler, Model N-251. For each presentation, the standard stimulus was presented first for $1 \mathrm{sec}$; after an interval of $1 \mathrm{sec}$, the comparison stimulus came on for 1 sec. There was a 4-sec interval between each pair of stimuli. Ss, in a sound-attenuated chamber, had a three-key response box, through which they indicated judgments of "higher," "equal," or "lower." Durations of the tones and intervals were controlled by Gerbrands "300" series millisecond clocks. All tones were at $60 \mathrm{~dB}$ SPL.

Each $S$ participated in two threshold determinations, involving 225 paired presentations each. The two determinations were separated by 1 week. The only difference between the two conditions was that in the anchor condition, the 4-sec interval between paired presentations of standard and comparison stimuli was filled by a $750-\mathrm{Hz}$ tone, in effect a low-tone anchor.

Of primary concern was the comparison of $I u$ for the two conditions. The mean Iu for the control condition was 14.4 ; for the anchor condition, it was $25.2(t=$ $2.68, p<.05$ ). Thus, introduction of the anchor did, as hypothesized, increase the $\mathrm{Iu}$.

A further inspection of the data revealed that the primary effect of the anchor was on the lower limen (L): the stimulus value midway between the last judgment of "equal" and the first judgment of "lower"). For the control condition, $L l$ was $1,484.3 \mathrm{~Hz}$; for the anchor condition, it was $1,477.6 \mathrm{~Hz}$. The difference of $6.7 \mathrm{~Hz}$ was highly significant $[t(10)=32.6, p<.001]$. Corresponding values for the upper limen (Lu) were $1,498.3$ for the control and 1,500.5 for the anchor; the difference was not significant $[t(10)=1.78, p>.05]$.

\section{DISCUSSION}

The results of both experiments appear to indicate two primary findings having to do with the perception of pitch. First, the introduction of, and relative emphasis on, a low-frequency tone interferes with pitch discrimination for other tones; second, the effect appears to be more pronounced for tones nearer in frequency to the anchor than for those more remote.

Table 1

Standard Deviations for Judgments of Each Series Tone

\begin{tabular}{lccc}
\hline & $\begin{array}{l}\text { Tone } \\
\text { (Hz) }\end{array}$ & Control & Anchor \\
\hline & 1000 & 1.58 & $\mathbf{3 . 7 7}$ \\
Group I & 1250 & 1.61 & 4.06 \\
& 1500 & 1.59 & $\mathbf{3 . 8 7}$ \\
& 1750 & 1.15 & $\mathbf{3 . 7 5}$ \\
& 2000 & .90 & $\mathbf{3 . 0 4}$ \\
& 2000 & 1.79 & $\mathbf{2 . 6 1}$ \\
Group I1 & 2250 & $\mathbf{2 . 1 4}$ & $\mathbf{2 . 4 1}$ \\
& 2500 & 2.04 & $\mathbf{3 . 3 9}$ \\
& 2750 & 1.38 & 2.25 \\
& 3000 & 1.15 & $\mathbf{3 . 2 0}$ \\
\hline
\end{tabular}


The first finding was supported by the lack of orderly slope for the anchored judgments shown in Fig. 1 , as opposed to the control functions which imply excellent discrimination. Although the findings of Experiment 1 may be affected by an assumed range curtailment under the anchor conditions, that explanation is likely to be partial at best when the findings of Experiment 2 are considered. The increase in Iu under the anchor condition in that experiment supports the contention of a more purely perceptual effect, although both perceptual and response factors may have been operating in Experiment 1 .

One concern 1 relating to Experiment 2 was that the tone-filled 4-sec interval of the anchor condition may have caused physiological adaptation, thereby increasing $\mathrm{Iu}$. Accordingly, a post hoc experiment using eight Ss, opposed a control (silent interval) condition to one in which the interval was filled with white noise, at $60 \mathrm{~dB}$ SPL. Contrary to the conjecture, Iu was significantly smaller under the white noise condition $(\bar{X}=9.4)$ than under the silent condition $(\bar{X} 16.4 ; t=3.2$, $p<.02$ ). It is therefore unlikely that adaptation accounted for the anchor effects in Experiment 2. An explanation for the constraining effect of white noise on Iu is conjectural at best. Two Ss reported that it appeared to "focus" attention on the paired tones, suggesting a possible analogue to a figure-ground enhancement.

Other studies of $\mathrm{AE}$, using different modalities (Adamson, 1967; Sarris, 1969), have found that anchors primarily tend to influence judgments of stimuli proximal to the anchor, the effect lessening with more remote stimuli. Experiment 1 grossly supports this finding, in that the difference in mean judgments between anchor and control conditions was more marked for Group I than for Group II. That the Ll in Experiment 2 was significantly lowered by the presence of the anchor provides more particular support to the finding, especially since the Lu was unaffected.

There was, apparently, no harmonic effect of the anchor. Figure 1 shows no effect on the anchor function at the first harmonic $(1,500 \mathrm{~Hz})$, an elevation at the second $(2,252 \mathrm{~Hz})$, and a possible depression at the third $(3,000 \mathrm{~Hz})$. Additionally, in the DL study, values about the standard stimulus equal to the first harmonic of the anchor were less affected in judgment than those closer to the anchor itself.

The reduction in discrimination associated with anchor effects has been found for judgmental dimensions other than pitch and in a variety of paradigms. Rogers (1941), using the method of single stimuli, found that for judgments of lifted weights and line inclination, ". . . as the anchoring stimulus recedes from the stimulus range, judgments become more difficult and uncertain, hesitant." The dependence of the position and, more importantly, width of the absolute scale on the remoteness of the anchor had also been shown earlier by Volkman (1936), whose Ss judged line inclination with anchors that were defined by instruction but not physically present.

Gravetter and Lockhead (1970) had Ss make absolute judgments of loudness under two conditions of stimulus range. Differentiation decreased with greater range, leading to their contention that variability due to the scale of judgments directly reflects the extent of physical range over which stimuli are presented. In their study, variability was measured for a subset of stimuli whose interstimulus distance was constant for the two range conditions, changes in range being accomplished by varying the distance of more extreme stimuli from the group being judged. Thus, the situation was one in which anchors were bidirectionally displaced from the series, and possibly compensatory in their effects on mean judgments, no differences in the latter being shown.

In a theory of intensity resolution relating to loudness scaling, Durlach and Braida (1969) refer to a "context-coding mode" in which $S$ related a given sensation to others engendered by stimuli presented in the same experiment. In this mode, "... the amount of noise depends on the width of the context, larger widths leading to greater noise." If one equates context width with the physical stimulus range, as Durlach and Braida do, then they, as well as Gravetter and Lockhead, predict a decrease in discriminability with the introduction of anchors. It is self-evident that the latter, particularly if judged in the same fashion as series stimuli, act as range extenders. Thus, the findings of the present study offer qualified support for the theoretical position outlined in the foregoing.

Although Attneave and Olson (1971) argue that pitch, being morphophoric, is inappropriately treated in conventional psychophysical fashion, they offered findings that could be interpreted, in part at least, by context width effects. In testing for transposition by reconstruction from a familiar three-note pattern in the $196-330-\mathrm{Hz}$ range, they found that variable error, while uniform for frequencies close to the original pattern doubled in the neighborhood of $5,000 \mathrm{~Hz}$ and increased by a factor of four at higher frequencies. They attributed the finding to the nonmusical character of higher frequencies or to a possible shift from a volley to a place mechanism of pitch. However, it could be due in part to the range effect.

Anchor effects appear to be at least two in number: a displacement of judgments for series stimuli, and an increased variability of judgment as an indicant of lessened discriminability. The former effect has traditionally been treated by concepts like adaptation level (Helson, 1964), which, according to the present findings, may deal appropriately only with restricted portions of the stimulus range. It is a truism that judgments of the weight of one or two b-bs are probably unaffected by hoisting a steamer trunk.

The effect on variability of judgment may be similarly limited. For example, reference to Table 1 indicates that increasing the stimulus range from 1,000 to $1,250 \mathrm{~Hz}$ (Group I) increases response variability as much as, or more than, a shift from 1,000 to $2,250 \mathrm{~Hz}$ (Group II), although it might be argued in either case that the anchor has effectively abolished pitch resolution.

\section{REFERENCES}

ADAMSON, R Anchor effect limits. Psychonomic Science, 1967, 9, 178-180. ATTNEAVE, F., \& OLSON, R. K. Pitch as a medium: A new approach to psychophysical scaling. American Journal of Psychology, 1971, 84, 147-166.

BEVAN, W \& PRITCHARD, J F The anchor effect and the problem of relevance in the judgment of shape. Journal of General Psychology, 1963, 69 , 147-161.

DURLACH，NＩ＆ BRAIDA， L D Intensity perception: I. Preliminary theory of intensity resolution. Journal of the Acoustical Society of America, 1969 46, 372-383.

GR AVETTER, F. J., \& LOCKHEAD, G. R. Criterial dispersions and the stimulus range. Proceedings, 78 th Annual Convention, APA, 1970, 41-42.

HELSON, H. Adaptation-level theory. New York: Harper \& Row, 1964.

ROGERS, $S$. The anchoring of absolute judgments. Archives of Psychology, 1941, 261, 5-42.

SARRIS, V. Anchor effects in psychophysics: Tests on the adaptation-level model. Paper given at $X I X$ International congress of Psychology, London, 1969.

VOLKMAN, J. The anchoring of absolute scales. Psychological Bulletin, 1936, 33 742.

\section{NOTE}

1. Suggested by Charles Eriksen in an editorial comment, as was the white noise control.

(Accepted for publication May 22, 1972.) 